\title{
Sexual Function and Quality of Life Among Postpartum Women: A Cross-Sectional Study
}

\author{
Nazanin Rezaei ${ }^{1}$, Fatemeh Janani ${ }^{1}$, Nasibeh Sharifi ${ }^{2}$, Fatemeh Omidi ${ }^{3}$, Arman Azadi $^{4^{*}}$
}

\begin{abstract}
Objectives: Sexual function plays an important role in strengthening the marital relationship. Sexual issues affect individuals' quality of life. This study aimed to investigate the association between the sexual function and the quality of life among postpartum women in Iran.

Materials and Methods: For this cross-sectional descriptive study, 380 postpartum women who had referred to 10 urban health care centers in the west of Iran were chosen. They were recruited using the randomized cluster sampling method. A checklist for socioindividual and maternal status of women, female sexual function index (FSFI) and SF-36 questionnaire were used for data collection. Descriptive and inferential statistics were used for data analysis via SPSS software.

Results: Most of the participants (76.3\%) suffered from sexual dysfunction. Sexual desire was the most prevalent sexual dysfunction (79\%). Moreover, the majority of the women achieved moderate scores in the subscales of the SF-36 questionnaire. The women with higher FSFI scores achieved higher scores in all SF-36 subscales. The Pearson correlation coefficient showed an appositive statistically significant linear correlation between the women's general health and all dimensions of the FSFI except for pain during sexual relationships. The logistic regression analysis revealed that sexual function was a protective factor against the reduction of the quality of life, because quality of life was higher among the women with a higher level of sexual function compared to those with a lower sexual function $(\mathrm{P}<0.05)$.

Conclusions: Sexual dysfunction in the postpartum period could adversely affect the women's quality of life. While sexual problems are very common in the postpartum period, they are not reported in most cases. Health care providers should design appropriate programs such as extended postpartum counseling for the women in the postpartum period.

Keywords: Quality of life, Sexual dysfunction, Postpartum, Childbirth, Delivery, Iran
\end{abstract}

\section{Introduction}

The promotion of women's health is one of the most important indicators of the health status of a nation (1). Sexual activities in the postpartum period and the provision of related advice are the important elements of the women's health care $(1,2)$. Out of $90 \%$ of the women who begin sexual activities 6 months after childbirth, $84 \%$ experience sexual problems (3). Appropriate sexual function can lead to the improvement of individuals' life conditions. On the contrary, sexual dysfunction can affect both parents' quality of life and their marital relationship, as sexual desire plays a key role in the individuals' health, quality of life, and overall welfare $(3,4)$. In many countries, sexual dysfunction is considered a taboo subject that negatively affects the quality of life and may cause mental disorders (5). Therefore, the postpartum period can be considered for the examination of health-related issues and sexual function (2). However, a small fraction of women (15\%) report sexual problems after childbirth or consultation with the healthcare team. According to previous studies, this can be due to a lack of information about the healthcare team or unwillingness to discuss this problem in postpartum care (6-8).

Consulting about sexual problems especially in Asian societies such as Iran have not been discussed generally and widely due to cultural and religious issues. Consequently, this may hinder providing appropriate services for the target group $(1,9)$.

The quality of life is a multi-dimensional phenomenon and is influenced by physical, psychological, emotional, social, sexual, and spiritual health factors (10). A few studies have been carried out on the relationship between the quality of life in the postpartum period and factors influencing it (11-14). Although sexual dysfunction is one of the factors that adversely affects women's quality of life in the postpartum period, the association between the sexual function and the quality of life has not been adequately studied $(1,2,12)$. Therefore, this study aimed to examine the association between sexual function and the quality of life in the postpartum period.

\section{Materials and Methods}

This cross-sectional study was conducted in 10 urban healthcare settings in a city in the west of Iran. The study 
group consisted of all the women who met the following criteria: less than 8 months had passed since childbirth, aged $\geq 18$ years old, childbirth at $38-42$ weeks of the gestational age, and the desire to participate in the study. Women with the following criteria were excluded from the study: any obstetric complications for the mother or baby at birth, postpartum depression, any disability or chronic illness, any type of surgery in the past 3 months for each spouse, and genital lesions that caused some problems in sexual intercourse. Sampling was performed using the cluster random sampling method. Initially, the city of data collection was divided into 5 geographic regions (center, north, south, east, and west). Next, 2 health care centers in each region were selected randomly. Each region was considered a cluster. Since the number of households covered by each healthcare center was different, proportional random sampling was used to determine the sample size in each center. After reviewing family health records, eligible women were identified and randomly selected to participate in the study. The aim and method of study were explained to the women. The participants were informed that collected data will be kept confidential and the women could withdraw from the study anytime without any effect on their care.

The sample size $(n=375)$ was calculated based on $\alpha=$ 0.05 and $\sigma=22$ (15). Given the possibility of the attrition of the samples, 400 women were selected randomly using the numbers assigned to the women's medical records. Lastly, 380 postpartum women were accepted to take part in the project and fill out the questionnaires (response rate $=95 \%)$.

A checklist examined the socio-demographic characteristics of the participants and was filled out based on the women's self-report. Demographic data consisted of age, number of livebirth, method of childbirth, occupation, medical history, education level, and history of divorce. The health-related quality of life (HRQOL) questionnaire was a multi-dimensional instrument and was validated by Montazeri et al(16) in Iran. It examined 8 aspects of the quality of life including physical function (10 items), general health (5 items), bodily pain ( 2 items), role limitations due to physical problems (4 items), vitality (4 items), role limitations due to emotional problems (3 items), mental health (5 items), and social function (2 items). The internal consistency of this instrument was assessed using the calculation of Cronbach's alpha coefficients in 8 dimensions that were reported as 0.77 0.90 (16).

The women's sexual activities in the previous month was studied using the female sexual function index (FSFI). This instrument was designed in 2000 by Rosen et al (17) and its reliability was assessed by previous studies (1820). This scale evaluated 6 domains of sexual function in the past month including sexual desire ( 2 cases), arousal (4 cases), lubrication (4 cases), orgasm (3 cases), sexual satisfaction (3 cases), and pain (3 cases). Moreover, the questions 1, 2, 15 and 16 were scored from 1 to 5 and other questions were scored from 0 to 5 . The zero score in each of these areas indicated no sexual activity in the previous month. The summation of the domains' scores was multiplied by the related coefficients (5). The range of the scores was reported as 2-36 and a lower score indicated a poorer sexual function. FSFI $\leq 28$ was considered sexual dysfunction. Furthermore, achieving less than 65 of the total score of each domain indicated sexual dysfunction in that domain. As a result, scores less than 3.9 in each 6 domains were considered sexual dysfunction (18).

The reliability and validity of this questionnaire were evaluated by other studies $(19,20)$. In terms of reliability and validity, the Farsi version of this instrument was assessed by Mohammadi et al and Fakhri et al $(21,22)$. The internal consistency of the questionnaire was also assessed using the Cronbach's alpha coefficient, that was reported as $0.72-0.90$.

Before data collection, the Ethics Committee affiliated with Ilam University of Medical Sciences, Iran, approved the study research protocol. The researchers referred to the health care centers from June 2014 to June 2015. After explaining the aim of the study, the women were asked to participate in individual interviews. Interviews were carried out by a female interviewer in a private place convenient to the participants. The women who agreed to take part in this study signed the written informed consent form prior to the study.

Face and content validity of the questionnaires were assessed by 10 faculty members affiliated with the university in which the authors worked. The final versions of the questionnaires were tested for reliability in a pilot study on 25 women in the postpartum period. The SPSS software version 15.0 was used for data analysis. Relationships between the women's sexual function and the quality of life were assessed by inferential statistics including independent $t$ tests, chi-square, Pearson correlation coefficient, and logistic regression as appropriate.

\section{Results}

In this study, 380 women participated, from which 93, 153, and 134 women filled out the questionnaires at 8-12 weeks, 3-5 months, and 6-8 months of the postpartum period, respectively. About half of the women $(n=202,53.2 \%)$ were primiparous and the method of childbirth in 233 (61.3\%) of the women was the caesarean section. The mean (SD) of the participants' age was 29.81(5.5) years. Most of the women were housewives (86.6\%) and almost half of them (44.7\%) had an academic education degree (Table 1). The rate of cesarean section was higher among primiparous women than those with higher incomes and the educated women. The mean (SD) of the total sexual function in the postpartum period was 22.24 (7.93). The majority of the women $(76.3 \%)$ suffered from sexual dysfunction in the postpartum period (the score of the sexual function $\leq 28$ ). 
Table 1. The Maternal and Socioeconomic Characteristics of the Women

\begin{tabular}{|c|c|}
\hline Characteristics & No. (\%) \\
\hline \multicolumn{2}{|l|}{ Age (y) } \\
\hline $15-25$ & $74(19.5)$ \\
\hline $25-35$ & $234(61.6)$ \\
\hline$>35$ & $72(18.9)$ \\
\hline \multicolumn{2}{|l|}{ Education (y) } \\
\hline$<12$ & $210(55.3)$ \\
\hline$>12$ & $170(44.7)$ \\
\hline \multicolumn{2}{|l|}{ Job } \\
\hline Housewife & $329(86.6)$ \\
\hline Employed & $51(13.4)$ \\
\hline \multicolumn{2}{|c|}{ Number of deliveries } \\
\hline $1-2$ & 338 (88.9) \\
\hline$>2$ & $42(11.1)$ \\
\hline \multicolumn{2}{|l|}{ Income } \\
\hline Fair & $252(66.3)$ \\
\hline Poor & $128(33.7)$ \\
\hline \multicolumn{2}{|l|}{ Type of delivery } \\
\hline NVD & $147(38.7)$ \\
\hline CS & $233(61.3)$ \\
\hline \multicolumn{2}{|l|}{ Breastfeeding } \\
\hline Yes & $339(89.2)$ \\
\hline No & $41(10.8)$ \\
\hline \multicolumn{2}{|c|}{ Time passed since birth (mon) } \\
\hline$<3$ & $128(33.7)$ \\
\hline$>3$ & $252(66.3)$ \\
\hline
\end{tabular}

Abbreviations: NVD, normal vaginal delivery; CS, caesarian section.

The most common sexual dysfunction in the postpartum period was a lack of sexual desire, which was reported by $79 \%$ of the women. No statistically significant relationship was reported between the method of childbirth and sexual dysfunction in the postpartum period. Furthermore, there were no statistically significant relationships between the women's level of education, income and occupation, and sexual dysfunction. The mean score of sexual function was significantly higher in the non-lactating women compared to the lactating ones $(P=0.006)$.

No statistically significant relationship was reported between the women's quality of life and the method of childbirth. The younger women had a better quality of life in vitality, social function and bodily pain domains $(P<0.05)$. The employed women significantly had a higher quality of life in vitality, mental health and physical function domains compared to the housewives $(P<0.05)$. Moreover, different aspects of quality of life and breastfeeding had no statistically significant relationships. The mean scores of quality of life of the women in the postpartum period based on the sexual function are shown in Table 2. The women with higher sexual function scores had significantly higher quality of life in all aspects. The Pearson correlation coefficient showed a positive significant relationship between general health and other domains of sexual function except for pain during sexual intercourse (Table 3).

Binary logistic regression analyses showed the
Table 2. The Participants' Scores in Eight Aspects of HRQoL According to Their Sexual Function

\begin{tabular}{|c|c|c|c|}
\hline \multirow[b]{2}{*}{ Subscales of the SF-36 } & \multicolumn{2}{|c|}{ Sexual Dysfunction } & \multirow[b]{2}{*}{$P$-value } \\
\hline & $\begin{array}{c}\text { Yes } \\
\text { Mean(SD) }\end{array}$ & $\begin{array}{c}\text { No } \\
\text { Mean(SD) }\end{array}$ & \\
\hline General health & $48.77(14.0)$ & $55.38(13.2)$ & $<0.001$ \\
\hline Vitality & $57.20(19.1)$ & $70.72(15.2)$ & $<0.001$ \\
\hline Mental health & $65.37(18.2)$ & 77.28 (15.9) & $<0.001$ \\
\hline Role emotional & $58.73(39.6)$ & $68.88(36.6)$ & 0.031 \\
\hline Social function & $65.64(22.1)$ & 78.33 (17.55) & $<0.001$ \\
\hline Bodily pain & $61.24(21.3)$ & $73.26(15.5)$ & $<0.001$ \\
\hline Role physical & $57.74(36.4)$ & $69.44(32.4)$ & 0.001 \\
\hline Physical function & $65.32(26.33)$ & $75.00(22.2)$ & 0.002 \\
\hline Physical HRQoL (total) & $57.74(16.57)$ & $68.27(14.84)$ & $<0.001$ \\
\hline Mental HRQoL (total) ${ }^{\mathrm{b}}$ & $61.74(19.3)$ & 73.80 (16.9) & $<0.001$ \\
\hline
\end{tabular}

a Physical HRQoL referred to mean score of $P F+B P+R P+G H$.

${ }^{b}$ Mental $\mathrm{HRQ}$ oL referred to mean score of $\mathrm{VT}+\mathrm{MH}+\mathrm{SE}+\mathrm{RE}$.

relationship between the dimensions of sexual function and the quality of life. The estimated odds ratios (OR) and $95 \% \mathrm{CI}$ of binary logistic regression analysis are reported in Table 4. According to the logistic regression analysis, the dimensions of sexual function including arousal, lubrication, orgasm, and sexual satisfaction were protective factors against the quality of life. The probability of poor physical and mental quality of life was lower among the women with higher sexual function scores compared to those with a lower sexual function. The women with high sexual desire had $31 \%$ lower odds of poor physical quality of life than those with lower sexual desire $(\mathrm{CI}=0.415-1.177, \mathrm{OR}=0.699)$. Likewise, the women with low pain had $16 \%$ lower odds of poor physical quality of life than those with high feeling of pain $(\mathrm{CI}=0.566-1.267, \mathrm{OR}=0.847)($ Table 4$)$.

\section{Discussion}

It was found that the most prevalent sexual dysfunction was the lack of sexual desire. In the studies of Shirvani et al and Boroumandfar et al in Iran, a reduction in sexual desire was reported for $40 \%$ of the women in postpartum period $(3,23)$. However, the findings of other studies in Iran showed that only $2.4 \%$ of the women reported postpartum sexual problems due to cultural and social beliefs (23). Additionally, healthcare providers lacked enough knowledge and time during consultation in the postpartum period, which caused insufficient advice regarding sexual problems in the postpartum period. The study of Cheng et al showed that most women preferred to discuss sexual issues with healthcare providers instead of their family or friends (24). The findings of previous studies showed that healthcare providers' education besides support by the family and spouses led to the improvement of sexual relationships and quality of life in the postpartum period $(25,26)$. Emerging various life changes after childbirth such as fatigue, anxiety and caring for the newborn negatively affected the women's sexual 
Table 3. The Correlation Between the Quality of Life Dimensions and the Sexual Function of the Women in the Postpartum Period

\begin{tabular}{|c|c|c|c|c|c|c|}
\hline \multirow{3}{*}{ FSFI Domains } & \multicolumn{6}{|c|}{ Subscales of the SF-36 } \\
\hline & \multicolumn{3}{|l|}{ Physical Health } & \multicolumn{3}{|l|}{ Mental Health } \\
\hline & General Health & Physical Function & Bodily Pain & Mental Health & Social Function & Vitality \\
\hline Sexual desire & $0.138^{* *}$ & 0.041 & $0.200 * *$ & $0.235^{* *}$ & $0.262^{* *}$ & $0.266^{* *}$ \\
\hline Arousal & $0.118^{*}$ & $0.104^{*}$ & $0.249 * *$ & $0.257^{* *}$ & $0.262^{* *}$ & $0.254^{* *}$ \\
\hline Lubrication & $0.123^{*}$ & $0.162 * *$ & $0.249 * *$ & $0.243^{* *}$ & $0.245^{* *}$ & $0.221^{* *}$ \\
\hline Orgasm & $0.163 * *$ & $0.154 * *$ & $0.267^{* *}$ & $0.248^{* *}$ & $0.250 * *$ & $0.241^{* *}$ \\
\hline Satisfaction & $0.210 * *$ & $0.112^{*}$ & $0.295^{* *}$ & $0.304 * *$ & $0.312^{* *}$ & $0.270^{* *}$ \\
\hline Pain & 0.070 & 0.065 & $0.209 * *$ & $0.135^{* *}$ & $0.180 * *$ & $0.197 * *$ \\
\hline
\end{tabular}

$* P<0.05, * * P<0.01$.

Table 4. The Relationship Between the Sexual Function and Quality of Life in the Postpartum Period Based on the Logistic Regression Analysis

\begin{tabular}{|c|c|c|c|c|c|c|}
\hline \multirow{2}{*}{ Variable } & \multicolumn{3}{|c|}{ Physical Quality of Life } & \multicolumn{3}{|c|}{ Mental Quality of Life } \\
\hline & OR & $95 \% \mathrm{Cl}$ & $P$ Value & OR & $95 \% \mathrm{Cl}$ & $P$ Value \\
\hline \multicolumn{7}{|l|}{ FSFI Domains } \\
\hline Sexual desire & 0.699 & $0.415-1.177$ & 0.178 & 0.713 & $0.423-1.203$ & 0.205 \\
\hline Arousal & 0.541 & $0.356-0.822$ & 0.004 & 0.441 & $0.288-0.675$ & $<0.001$ \\
\hline Lubrication & 0.401 & $0.264-0.609$ & $<0.001$ & 0.502 & $0.332-0.759$ & 0.001 \\
\hline Orgasm & 0.367 & $0.240-0.562$ & $<0.001$ & 0.389 & $0.255-0.594$ & $<0.001$ \\
\hline Sexual satisfaction & 0.411 & $0.260-0.649$ & $<0.001$ & 0.458 & $0.291-0.720$ & 0.001 \\
\hline Pain & 0.847 & $0.566-1.267$ & 0.418 & 0.676 & $0.451-1.014$ & 0.058 \\
\hline Total & 0.409 & $0.235-0.711$ & 0.002 & 0.350 & $0.197-0.620$ & $<0.001$ \\
\hline
\end{tabular}

Abbreviations: FSFI, Female sexual function index, Poor quality of life $=1$, Good quality of life $=0$.

life and quality of life $(2,6,27)$; though, the women's sexual problems improved gradually after childbirth $(23,28)$. However, the findings of some studies showed that the women reported some degrees of sexual dysfunction one year after childbirth $(23,27)$. Therefore, it is recommended to pay more attention to the sexual problems of women in the postpartum period that consequently can improve women's quality of life. The findings of the present study also showed that most women had a moderate level of quality of life, which was consistent with the findings of Bahrami et al in Iran (29).

In the present study, there was a statistically significant relationship between the women's sexual function and quality of life. Moreover, in this study, sexual function played a protective role for the quality of life. The women with high sexual function had $60 \%$ and $65 \%$ less poor physical and mental quality of life than those with a low sexual function. These findings are consistent with the results of Naeinian et al and Lau et al in Iran and China, respectively $(30,31)$.

There was a positive statistically significant relationship between the mental aspects of quality of life such as mental health, social function and vitality and different aspects of the sexual function. Lau et al believed that sexual problems in women were more related to their mental health. Sexual health and quality of life were also associated together (31, 32). Consequently, a low sexual desire as a main problem was reported by the women in this study, which led to low sexual satisfaction, low quality of marital relationship, and low quality of life. Satisfaction with sexual relationships and sufficient sexual desire were the most important parts of the marital relationship, which could affect the quality of life in social, psychological and physical aspects $(33,34)$.

\section{Limitations}

Data about the women's quality of life and the sexual function before childbirth was not collected. Therefore, no evidence is available whether sexual problems occurred in the postpartum period or in the childbirth. Some other possible confounding factors might also have affected the validity of the findings.

\section{Conclusions}

Postpartum sexual dysfunction can affect the women's quality of life. As postpartum care is limited to 6 weeks after childbirth, the duration of providing postpartum care should be reconsidered, for instance one year after the childbirth. In addition, the content of postpartum care should be revised with an emphasis on sexual problems. It is also recommended to examine the effectiveness of such programs on the women's quality of life in societies with different cultures. Health care providers should design appropriate programs such as extended postpartum counseling for the women in the postpartum period.

\section{Ethical Issues}

This paper was derived from a research project approved in Research Council of Ilam University of Medical Sciences 
under the code of ethics: EC/93/H/259.

\section{Conflict of Interests}

No conflict of interests is declared by the authors in this study.

\section{Financial Support}

This study was funded by Ilam University of Medical Sciences, Ilam, Iran.

\section{Acknowledgments}

The authors are grateful to all the individuals who assisted us in the completion of this study including the research deputy of Ilam University of Medical Sciences, Iran, and the women who participated in this study.

\section{References}

1. Radziah M, Shamsuddin K, Jamsiah M, et al. Early resumption of sexual intercourse and its determinants among postpartum Iban mothers. Int J Reprod Contracept Obstet Gynecol. 2013;2(2):124-129. doi:10.5455/23201770.ijrcog20130603

2. Abdool Z, Thakar R, Sultan AH. Postpartum female sexual function. Eur J Obstet Gynecol Reprod Biol. 2009;145(2):133-137. doi:10.1016/j.ejogrb.2009.04.014

3. Boroumandfar K, Rahmati MG, Farajzadegan Z, Hoseini $\mathrm{H}$. Reviewing sexual function after delivery and its association with some of the reproductive factors. Iran J Nurs Midwifery Res. 2010;15(4):220-223.

4. Mohammadalizadeh Charandabi S, Rezaei N, Hakimi S, et al. Quality of life of postmenopausal women and their spouses: a community-based study. Iran Red Crescent Med J. 2015;17(3):e21599. doi:10.5812/ircmj.21599

5. Safarinejad MR. Female sexual dysfunction in a populationbased study in Iran: prevalence and associated risk factors. Int J Impot Res. 2006;18(4):382-395. doi:10.1038/ sj.ijir.3901440

6. Botros SM, Abramov Y, Miller JJ, et al. Effect of parity on sexual function: an identical twin study. Obstet Gynecol. 2006;107(4):765-770. doi:10.1097/01. aog.0000207677.03235.76

7. Nicolosi A, Buvat J, Glasser DB, Hartmann U, Laumann EO, Gingell C. Sexual behaviour, sexual dysfunctions and related help seeking patterns in middle-aged and elderly Europeans: the global study of sexual attitudes and behaviors. World J Urol. 2006;24(4):423-428. doi:10.1007/ s00345-006-0088-9

8. Ho TM, Fernandez M. Patient's sexual health: do we care enough? J Ren Care. 2006;32(4):183-186.

9. Ghanbarzadeh N, Nadjafi-Semnani M, Ghanbarzadeh MR, Nadjfai-Semnani A, Nadjfai-Semnani F. Female sexual dysfunction in Iran: study of prevalence and risk factors. Arch Gynecol Obstet. 2013;287(3):533-539. doi:10.1007/ s00404-012-2604-z

10. Ribeiro SG, Symon AG, Lessa PR, et al. Translation and cultural adaptation of the Mother-Generated Index into Brazilian Portuguese: A postnatal quality of life study. Midwifery. 2015;31(7):735-741. doi:10.1016/j. midw.2015.03.009

11. Torkan B, Parsay S, Lamyian M, Kazemnejad A, Montazeri
A. Postnatal quality of life in women after normal vaginal delivery and caesarean section. BMC Pregnancy Childbirth. 2009;9:4. doi:10.1186/1471-2393-9-4

12. Van der Woude DA, Pijnenborg JM, de Vries J. Health status and quality of life in postpartum women: a systematic review of associated factors. Eur J Obstet Gynecol Reprod Biol. 2015;185:45-52. doi:10.1016/j.ejogrb.2014.11.041

13. Prick BW, Bijlenga D, Jansen AJ, et al. Determinants of health-related quality of life in the postpartum period after obstetric complications. Eur J Obstet Gynecol Reprod Biol. 2015;185:88-95. doi:10.1016/j.ejogrb.2014.11.038

14. Trivino-Juarez JM, Nieto-Pereda B, Romero-Ayuso D, et al. Quality of life of mothers at the sixth week and sixth month post partum and type of infant feeding. Midwifery. 2016;34:230-238. doi:10.1016/j.midw.2015.11.003

15. Mohammad-Alizadeh-Charandabi S, Rezaei N, Hakimi S, Montazeri A. Predictors of health-related quality of life in postmenopausal women: a population-based study. J Caring Sci. 2012;1(4):201-208. doi:10.5681/jcs.2012.028

16. Montazeri A, Goshtasebi A, Vahdaninia M, Gandek B. The Short Form Health Survey (SF-36): translation and validation study of the Iranian version. Qual Life Res. 2005; 14(3):875-882.

17. Rosen R, Brown C, Heiman J, et al. The Female Sexual Function Index (FSFI): a multidimensional selfreport instrument for the assessment of female sexual function. J Sex Marital Ther. 2000;26(2):191-208. doi:10.1080/009262300278597

18. Meston CM. Validation of the Female Sexual Function Index (FSFI) in women with female orgasmic disorder and in women with hypoactive sexual desire disorder. J Sex Marital Ther. 2003;29(1):39-46. doi:10.1080/713847100

19. Meston CM, Derogatis LR. Validated instruments for assessing female sexual function. J Sex Marital Ther. 2002;28 Suppl 1:155-164. doi:10.1080/00926230252851276

20. Wiegel M, Meston C, Rosen R. The female sexual function index (FSFI): cross-validation and development of clinical cutoff scores. J Sex Marital Ther. 2005;31(1):1-20. doi:10.1080/00926230590475206

21. Fakhri A, Pakpour AH, Burri A, Morshedi H, Zeidi IM. The Female Sexual Function Index: translation and validation of an Iranian version. J Sex Med. 2012;9(2):514-523. doi:10.1111/j.1743-6109.2011.02553.x

22. Mohammadi KH, Heydari M, Faghihzadeh S. Validity ofthe Persian version of the female sexual function index (FSFI) as an indicatorof women's sexualfunction. Payesh. 2008;7(3):269-278.

23. Shirvani MA, Nesami MB, Bavand M. Maternal sexuality after child birth among Iranian women. Pak J Biol Sci. 2010;13(8):385-389.

24. Cheng CY, Fowles ER, Walker LO. Continuing education module: postpartum maternal health care in the United States: a critical review. J Perinat Educ. 2006;15(3):34-42. doi:10.1624/105812406x119002

25. Akyn B, Ege E, Kocodlu D, Demiroren N, Yylmaz S. Quality of life and related factors in women, aged 15-49 in the 12-month post-partum period in Turkey. J Obstet Gynaecol Res. 2009;35(1):86-93. doi:10.1111/j.14470756.2008.00870.x

26. Olsson A, Robertson E, Falk K, Nissen E. Assessing women's sexual life after childbirth: the role of the postnatal check. Midwifery. 2011;27(2):195-202. doi:10.1016/j. midw.2009.04.003 
27. Holanda JBL, Abuchaim ESV, Coca KP, Abrao A. Sexual dysfunction and associated factors reported in the postpartum period. Acta Paul Enferm. 2014;27(6):573-578. doi:10.1590/1982-0194201400093.

28. Barrett G, Pendry E, Peacock J, Victor C, Thakar R, Manyonda I. Women's sexual health after childbirth. BJOG. 2000;107(2):186-195.

29. Bahrami N, Karimian Z, Bahrami S, Bolbolhaghighi N. Comparing the postpartum quality of life between six to eight weeks and twelve to fourteen weeks after delivery in iran. Iran Red Crescent Med J. 2014;16(7):e16985. doi:10.5812/ircmj.16985

30. Naeinian MR, Shaeiri MR, Hosseini FS. General health and quality of life in patients with sexual dysfunctions. Urol J. 2011;8(2):127-131.

31. Lau JT, Kim JH, Tsui HY. Prevalence of male and female sexual problems, perceptions related to sex and association with quality of life in a Chinese population: a populationbased study. Int J Impot Res. 2005;17(6):494-505. doi:10.1038/sj.ijir.3901342

32. Malouf MA, Inman AG, Carr AG, Franco J, Brooks LM. Health-related quality of life, mental health and psychotherapeutic considerations for women diagnosed with a disorder of sexual development: congenital adrenal hyperplasia. Int J Pediatr Endocrinol. 2010;2010:253465. doi:10.1155/2010/253465

33. Baldwin DS. Depression and sexual dysfunction. Br Med Bull. 2001;57:81-99.

34. Rezaei N, Azadi A, Zargousi R, Sadoughi Z, Tavalaee Z, Rezayati M. Maternal Health-Related Quality of Life and Its Predicting Factors in the Postpartum Period in Iran. Scientifica (Cairo). 2016;2016:8542147. doi:10.1155/2016/8542147

(c) 2018 The Author (s); This is an open-access article distributed under the terms of the Creative Commons Attribution License (http://creativecommons.org/licenses/by/4.0), which permits unrestricted use, distribution, and reproduction in any medium, provided the original work is properly cited. 